\title{
Selected Abstracts from the Wilderness Medical Society's 2007 Annual Scientific Meeting
}

Improving Short-Term Medical Volunteer Work Abroad

Introduction.-Medical providers from developed (wealthy) countries often participate in short-term medical volunteer projects in developing countries. Various health and development experts have raised the concern that such work has the potential to be harmful to its recipient communities. The medical literature contains little research into the impact of such volunteer work, and even less which takes into account the perspective of recipient communities.

Objectives.-To determine the perception of short-term medical volunteer work from key informants in recipient communities in order to assist planners and/or volunteers of shortterm medical projects in improving their volunteer work abroad. This study sought to answer the following questions: Does short-term medical volunteer work address selected healthcare needs of local communities? What are the potential advantages and disadvantages of this type of work? How can short-term medical volunteer work be improved?

Methods.- The researchers conducted in-depth, semi-structured interviews with 72 individuals deemed key informants in Guatemala, Central America. The respondents included Guatemalan healthcare providers, foreign medical providers, Guatemalan health authorities, individuals working on health projects, and parents of children treated by a short-term volunteer group. These interviews were uploaded, coded and annotated using Atlas.ti (Scientific Software Development $\mathrm{GmbH}$, Berlin) to identify recurrent themes from the interviews. These themes were then analyzed and grouped into the following topics: Need for short-term medical volunteer work; dependence; burden on host community; and coordination.

Results.-It was commonly acknowledged that there is a need for increased access to medical care in Guatemala. An overwhelming majority of our interviewees believed that short-term medical volunteers were in the position to make an impact on improving that access to care in communities where they serve. Appropriate patient selection and attention to the payment system were most often cited as the means to avoid creating dependence on foreign aid and to increase the sustainability of volunteer efforts. Coordination with and respect for the local healthcare providers was the most frequently mentioned suggestion for improvement of short-term medical volunteer work.

Conclusion.- It is widely perceived that short-term volunteer groups do have the potential to address real needs of their recipient groups. However, insufficient understanding of existing healthcare resources and needs and inadequate coordination with local providers may result in perceived harm to the recipient community. More cooperation with local organizations and providers is likely to improve the perceptions and the actual health impact of short-term medical groups.

Heidi Green, BS

Tyler Green, BA

Andrew Kestler, MD, MBA

Jean Scandlyn, $\mathrm{PhD}$

Denver, CO, USA

\author{
Oxygen Saturation Does Not Predict Acute Mountain \\ Sickness in Individuals Climbing Mt Rainier
}

Introduction.-Acute mountain sickness (AMS) remains a common debilitating condition in visitors to altitude. We have previously shown at our study location that oxygen saturation $\left(\mathrm{SpO}_{2}\right)$ fails to correlate with the presence of AMS. It is unclear if $\mathrm{SpO}_{2}$ can reliably predict the future incidence of AMS with any degree of confidence.

Objectives.-To observe if oxygen saturation $\left(\mathrm{SpO}_{2}\right)$ subsequently predicts AMS in individuals attempting to climb higher on Mt Rainier.

Methods.-We prospectively observed 45 participants who were climbing Mt Rainier, WA. Co-synchronous resting pulse and saturation data were obtained using a finger pulse oximeter after arrival at altitude $(3078 \mathrm{~m})$. The next day all individuals attempted to climb higher to the summit of Rainier (4392 m). Upon return, subjects submitted questionnaire-based surveys assessing symptoms of AMS during summit attempts.

Results.- Twenty individuals (45\%) subsequently suffered from AMS. Pulse oximetry was not significantly associated with subsequent incidence of AMS $(P>.05$, backwards stepwise logistical regression). Mean oxygen saturations for those individuals who subsequently suffered from AMS (92 $\pm 3 \%)$ were not significantly different from those individuals who were asymptomatic $(91 \pm 2.8 \%, P>.05)$.

Conclusion.-We conclude that while pulse oximetry is a useful and readily available physiological tool, $\mathrm{SpO}_{2}$ fails to reliably predict AMS in subjects attempting to climb to higher altitudes.

\author{
Terry R. O'Connor, MD \\ Portland, OR, USA \\ Phil Bickler, MD \\ Gerald Dubowitz, MD \\ San Francisco, CA, USA
}

A Prospective Observational Study of Optic Nerve Sheath Diameter and Acute Mountain Sickness in Individuals Climbing Mt Everest

Introduction.-Processes leading to acute mountain sickness (AMS) are poorly understood. It has been hypothesized that elevated intracranial pressure at altitude may be a contributing factor. Optic nerve sheath diameter (ONSD), a correlate of intracranial pressure (ICP), has been previously assessed at altitude. Prior study has also demonstrated ONSD measurement greater than $5 \mathrm{~mm}$ to have high sensitivity in detecting cranial computed tomographic findings of elevated ICP.

Objectives.-To evaluate changes in ONSD in individuals climbing to high altitude and to determine whether ONSD greater than $5 \mathrm{~mm}$ can accurately predict symptoms of AMS.

Methods. - This was a prospective, blinded observational study on subjects who were climbing Mt Everest. The ONSD was measured $3 \mathrm{~mm}$ behind the globe of the eye using an 8- 
$\mathrm{MHz}$ curved array transducer in longitudinal and transverse planes bilaterally. ONSD was assessed at low altitude (Kathmandu, approximately $1219 \mathrm{~m}$ ) and then again for all subjects after arrival at Advanced Base Camp (6401 m). AMS was assessed using Lake Louise Score (LLS) at $6400 \mathrm{~m}$.

Results.-Of the 23 subjects, 13 had symptoms of AMS (LLS $\geq 2$ with headache). Mean ONSD at low altitude (4.22 $\mathrm{mm}, 95 \%$ confidence interval $[\mathrm{CI}] 3.93-4.51)$ was significantly different from mean ONSD at Advanced Base Camp (5.35 $\mathrm{mm}, 95 \% \mathrm{CI} 4.97-5.73, P<.05)$. The sensitivity of ONSD of $5.00 \mathrm{~mm}$ or more in detecting AMS was $100 \%$, and specificity was $30 \%$. With an incidence of AMS of $57 \%$, the positive predictive value of ONSD of $5.00 \mathrm{~mm}$ or more was $65 \%$, and the negative predictive value was $30 \%$.

Conclusion.- - In our study, we demonstrated a significant increase in ONSD in individuals after ascent to altitude. This suggests that extreme altitude exposure results in elevated ICP. Interestingly, even though nearly all subjects had evidence of elevated ICP, this finding was not specific for the diagnosis of AMS.

Terry R. O’Connor, MD Alfredo Sabbaj, MD Portland, OR, USA

\section{Increased Intraocular Pressure Measured by Portable Tonometry is a Specific Indicator of High Altitude Cerebral Edema}

Introduction.-Acute mountain sickness (AMS) and high altitude cerebral edema (HACE) are significant causes of morbidity and mortality in visitors to high altitude regions. Because high altitude illness includes a spectrum of symptomatology, recognition and diagnosis can be difficult, particularly in settings far from modern technological diagnostic aids, such as computed tomography. Early diagnosis of HACE may mean the difference between life and death; a delay in diagnosis can result in progression of symptoms and death from intracranial hypertension. A light and portable tool to help clinicians properly distinguish HACE from other causes of headache at altitude could aid in the quick diagnosis and treatment of this deadly disorder.

Objectives.-This prospective study investigates the utility of a portable tonometer to aid in the differentiation of benign causes of headache at altitude from HACE and AMS by measurement of intraocular pressure, an indirect measure of intracranial pressure.

Methods.-Patients complaining of headache and asymptomatic controls who had both recently ascended from $2800 \mathrm{~m}$ to $4280 \mathrm{~m}$ underwent ocular tonometry at the Himalayan Rescue Association Clinic in Pheriche Nepal. Subjects were scored for the LLSR (Lake Louise Self-Report), LLF (Lake Louise Functional) and LLCA (Lake Louise Clinical Assessment).

Results.-Increased intraocular pressure (IOP) correlated with the diagnosis of HACE and AMS. Mean IOP of controls was 15.5, benign headache 18.3, AMS 20.5 and HACE 29.2, a significant increase. An IOP of 31 had a $97 \%$ specificity for HACE $(P<.001)$.
Conclusion.-A portable tonometer is an accurate diagnostic tool to aid clinicians with the diagnosis of AMS and HACE.

\author{
Kelly J. Casperson, MD \\ Denver, CO, USA \\ Luanne Freer, MD \\ Everest Base Camp, Nepal
}

\section{The Role of Brain Water Diffusion in Acute Mountain Sickness (AMS)}

Introduction.-The pathophysiology of acute mountain sickness (AMS) remains unexplained. In high altitude cerebral edema, considered a late-form of AMS, there is marked vasogenic edema. Considerable controversy currently surrounds the role of brain water diffusion in the pathophysiology of AMS. Two recent papers suggest that restricted diffusion may predominate in $\mathrm{AMS}^{1,2}$; but vasogenic edema would be associated with unrestricted diffusion.

Objective.-We sought to clarify the role of water diffusion in AMS by applying diffusion tensor imaging (DTI) before and after high altitude exposure.

Methods.-We exposed 22 healthy volunteers to simulated high altitude in a hypobaric chamber $(\mathrm{Pb}=430 \mathrm{~mm} \mathrm{Hg})$ for 10 hours. Fourteen of the 22 developed AMS (headache plus nausea, dizziness or lightheadedness) with a Lake Louise AMS score $>3$. Each volunteer completed 3-Tesla magnetic resonance imaging while breathing hypoxic gas before (PRE) and after (POST) altitude exposure. From DTI we computed fractional anisotropy (FA) and mean diffusivity (MD).

Results.-In AMS, we observed lower FA and elevated MD in white matter, both PRE and POST altitude $(P<.05$; FA: AMS PRE $0.3879 \pm 0.0135$, POST $0.3879 \pm 0.0135$ vs NoAMS PRE $0.3879 \pm 0.0135$ and POST $0.3895 \pm 0.0124$; MD: AMS PRE $0.1727 \times 10^{-3} \pm 0.0036 \times 10^{-3}$, POST $0.1727 \times$ $10^{-3} \pm 0.0041 \times 10^{-3}$ vs No-AMS PRE $0.1683 \times 10^{-3} \pm$ $0.0018 \times 10^{-3}$ and POST $0.1694 \times 10^{-3} \pm 0.0024 \times 10^{-3}$ ). This pattern suggests a breakdown in cellular integrity, with increasing unrestricted water diffusion.

Discussion.--In AMS DTI revealed greater unrestricted water diffusion, both at PRE and POST altitude. This finding is suggestive of vasogenic edema, but the group differences were small and did not increase as symptoms worsened from PRE to POST. Equally important, no evidence was obtained to support a role for restricted diffusion (intracellular edema). Thus, we conclude that AMS is associated with a small elevation in vasogenic edema, with no evidence of intracellular edema. However, the DTI differences did not enlarge with worsening

1 Kallenberg K, Bailey DM, Christ S, et al. Magnetic resonance imaging evidence of cytotoxic cerebral edema in acute mountain sickness. J Cereb Blood Flow Metab. 2007;27:1064-1071.

2 Schoonman GG, Sandor PS, Nirkko AC, et al. Hypoxia-induced acute mountain sickness is associated with intracellular cerebral edema: a 3 T magnetic resonance imaging study. J Cereb Blood Flow Metab. 2007; May 23; Epub ahead of print. 
of AMS symptoms and thus their importance in AMS remains questionable.

Kelly Brown, BS

Andrew Subudhi, PhD

Vaughn Browne, MD, PhD

Robert Roach, PhD

Aurora, CO, USA

Steve Altobelli, $\mathrm{PhD}$

Arvind Caprihan, $\mathrm{PhD}$

Albuquerque, NM, USA

Intermittent Hypoxia and Chemoresponse During Rest and Exercise

Introduction.-Intermittent hypoxia $(\mathrm{IH})$ consists of bouts of hypoxic exposure interspersed with normoxic intervals. One of its potential applications would include pre-acclimatization for altitude exposure. The optimal IH protocol for augmenting ventilatory response is unknown.

Objectives.-The purpose of this study was to assess the effects of 2 different protocols of IH on both resting and exercise chemoresponse in humans.

Methods.-Ten males underwent two 7-day poikilocapnic IH protocols. The long-duration IH (LDIH) protocol consisted of daily 60-minute exposures to normobaric $12 \% \quad \mathrm{O}_{2}$. The short-duration (SDIH) protocol comprised twelve 5-minute bouts of normobaric $12 \% \mathrm{O}_{2}$, separated by 5 -minute bouts of room air, daily. Resting variables included isocapnic hypoxic ventilatory response (HVR), hypercapnic ventilatory response (HCVR), $\mathrm{CO}_{2}$ threshold and $\mathrm{CO}_{2}$ sensitivity (by the modified Read rebreathing method). Exercise variables included minute ventilation, oxygen saturation, and hyperoxic and hypercapnic ventilatory response (in both hypoxia and normoxia).

Results.-After the third IH exposure, HVR had increased by $77 \%( \pm 24 \%)$ and $67 \%( \pm 17 \%)$ for LDIH and SDIH, respectively, and then remained unchanged for the duration of the protocol. The time constants of the exponential fit for the rise in HVR were 1.41 and 1.03 (LDIH and SDIH, respectively). The HCVR was increased and the $\mathrm{CO}_{2}$ threshold was significantly reduced (left-shift) following $\mathrm{IH} . \mathrm{CO}_{2}$ sensitivity was unchanged. $\mathrm{IH}$ also caused significant increases in response to hyperoxia during exercise. During hypoxic exercise, submaximal oxygen saturation and peak power were both increased. There were no differences between the $2 \mathrm{IH}$ protocols for any of the measures.

Conclusion.-A 7-day IH protocol caused a rapid increase in HVR and increased HCVR at rest and caused a left-shift in the resting $\mathrm{CO}_{2}$ threshold. During exercise, $\mathrm{IH}$ led to an increase in the hypoxic drive and an improvement in oxygen saturation. SDIH was no different from LDIH at effecting these changes.

Michael S. Koehle, MD, PhD

A. William Sheel, $\mathrm{PhD}$

William K. Milsom, PhD

Donald C. McKenzie, MD, PhD

Vancouver, BC, Canada
The Australian Funnel-Web Spider (Hadronyche infensa): A Case Report and Clinical Review

Australia is recognized as home to many dangerous creatures including venomous snakes, poisonous aquatic life and deadly crocodiles. Less well-known internationally are the species of Australian venomous spiders. The funnel-web spider (Hadronyche infensa) is arguably the most venomous spider in the world. Envenoming following a bite from the Australian funnel-web spider can trigger a catecholamine storm with potentially lethal consequences. Prior to the development of specific funnel-web spider antivenom, 13 confirmed deaths from funnel-web spider envenomation had been recorded in Australia. Distributed along the east coast of Australia, the usual habitat of funnel-web spiders is bush and moist forest regions. Changes in geographical distribution over the past few decades have lead to funnel-web spiders being detected in urban locations. The implication of this geographical redistribution is to increase the risk of clinically significant envenoming to humans. We present a case of funnel-web spider bite requiring antivenom treatment and provide a clinical update on this uniquely Australian toxinological emergency.

David J. Rosengren, MBBS, FACEM Sean P. Rothwell, MBBS, FACEM Queensland, Australia

\section{Severe Exercise-Associated Hyponatremia on the Kokoda Trail, Papua New Guinea}

Introduction.-Exercise-associated hyponatremia is the most common medical complication of ultradistance exercise and is usually caused by excessive hypotonic fluid intake. We report a case of severe hyponatremia in a healthy male trekking the Kokoda Trail, in the remote Southern Highlands of Papua New Guinea.

Case Report.-A 43-year-old male collapsed and had a generalized seizure in the afternoon of the third day of a guided trek. He was evacuated the following morning and found to have a serum sodium of $107 \mathrm{mmol} / \mathrm{L}$ on arrival to the hospital.

Discussion.- - The case highlights that a high index of suspicion is required to identify patients with exercise-associated hyponatremia. Early diagnosis and appropriate management is critical to avoid the potentially fatal consequences of severe hyponatremia. The diagnosis and treatment of exercise-associated hyponatremia is particularly challenging in the remote Papua New Guinea jungle. Education of trek leaders, medics and trekkers in appropriate preventative measures and rapid treatment of exercise-associated hyponatremia is essential to avoid recurrence of this life-threatening condition.

Sean P. Rothwell, MBBS, FACEM David J. Rosengren, MBBS, FACEM Queensland, Australia 


\section{Injuries and Medical Resources Available During Endurance Mountain Bike Events}

Introduction.-Endurance mountain bike events have grown in popularity. The nature of endurance races poses new and different medical problems in addition to the problems normally seen with shorter distance events. There are currently no standardized requirements for medical coverage for these events and no analysis of common injuries has been reported.

Objective.-To identify common injuries sustained during endurance mountain bike events and to review the medical resources available and utilized during the event.

Methods.-A survey looking at the riders' experience, previous injuries or pre-existing medical conditions as well as basic demographic information was completed by 334 of 606 participants at 4 endurance mountain bike events. There was also an additional survey to be completed by injured riders looking at the injury and the treatment received and if the injury resulted in the rider withdrawing from the race.

A survey was done of the medical personnel at the event looking at the number of medical providers and their level of medical training, supplies, and proximity to local hospitals.

Results.-There were 9 riders who sought medical attention, with 5 of the riders sustaining injuries that ended their participation. There were 10 riders who reported injuries that were not race ending, with only 6 of these riders seeking medical attention for these injuries. The reported injuries were abrasions ( 8 riders), lacerations ( 3 riders), head injuries (2 riders), blisters ( 2 riders), eye injury (dust in eye) (1 rider) and aggravation of previous injury (1 rider). There were no fatalities. The medical resources ranged from Red Cross volunteers with a first aid kit to an ambulance and personnel from a local EMS agency. The medical services provided consisted primarily of cleaning and bandaging wounds. One of the riders went on his own to seek medical attention at a hospital for repair of a laceration. There was no medical evacuation of a rider during the events.

Conclusions.-There were few race-ending injuries and the majority of injured riders did not seek medical care. Riders may have ignored their injuries, or the injuries were treated by themselves or a teammate. The riders may have been concerned with the injury ending their race or they could not find medical care at the event. There was no consensus or standardized format for providing medical support for the events. Without clear guidelines for medical care, it is difficult to persuade riders to stop racing and seek medical care for what may ultimately prove to be a minor injury. We are using the information obtained from this event to help us develop more effective survey tools and to develop a guideline for minimum medical resources at each event.

Stephanie Lareau, MS III Henderson D. McGinnis, MD Winston-Salem, NC, USA

\section{Erectile Dysfunction Therapy: Back Country Implications and Risks}

Erectile dysfunction (ED) is a common problem, with 20 to 30 million American men affected in varying degrees. Beyond this some therapies, particularly oral medication, are being employed for enhanced performance by younger men with normal sexual function. Advances and improved understanding of erections have elucidated the physiologic mechanisms of penile smooth muscle tissue relaxation. This has resulted in invasive and non-invasive treatments for patients with varying degrees of sexual dysfunction. Particular awareness of the risks and side effects of these therapies and their management in a wilderness environment is a necessary part in planning a safe back country experience.

The awareness, diagnosis, and treatment of erectile dysfunction have expanded since the introduction of sildenafil citrate (Viagra ${ }^{(i x)}$ in 1998. Sildenafil is a phosphodiasterase-5 (PDE$5)$ inhibitor that increases the relaxation of penile smooth muscle and results in erections. Two additional PDE-5 inhibitors (vardenafil and tadalafil) became available subsequently and are in common use. Other treatments are in development.

Erectile dysfunction therapy, invasive and non-invasive, and new developments in treating erectile dysfunction will be discussed. The presentation will cover the risks and side effects of therapy and emphasize precautions for use and management of problems in a back country environment. The potential impact in a wilderness environment will be addressed.

\section{Torrence M. Wilson, MD Rochester, MN, USA}

\section{Elevated Cerebral Blood Flow (CBF) in Subjects with Acute Mountain Sickness (AMS) at 5421 m}

Background.-In spite of several previous investigations, the role of cerebral blood flow (CBF) in the etiology of acute mountain sickness (AMS) remains unclear.

Objective: To clarify the role of CBF in AMS we studied cerebral vasoreactivity to acute ascent to high altitude, hyperventilation and oxygen breathing in AMS subjects and controls.

Methods.-Twenty-seven participants traveled from sea level to La Paz, Bolivia (3500 m), where they spent 4 nights and were then transported to Chacaltaya $(5421 \mathrm{~m}$, barometric pressure $[\mathrm{PB}] \sim 410 \mathrm{~mm} \mathrm{Hg}$ ) and studied within 24 hours. We measured Lake Louise AMS scores, non-invasive continuous mean arterial blood pressure (MAP, mm Hg; Colin tonometer), $\mathrm{CBF}$ velocity $(\mathrm{CBFv})$ via transcranial Doppler (DWL X2), and arterial oxygen saturation levels $\left(\mathrm{SPO}_{2}\right.$; Criticare $503+$ ). Measurements were made during spontaneous breathing (SB), paced respirations at 15 breaths/minute (HVE), and return to SB while on $100 \% \mathrm{O}_{2}\left(\mathrm{SB}-\mathrm{O}_{2}\right)$.

Results.-Of the 27 subjects studied, 19 developed AMS and 8 remained essentially free of AMS symptoms (Lake Louise AMS score: $4.5 \pm 0.3$ AMS + vs $0.25+0.2$ in AMS - ). $\mathrm{AMS}+$ had lower $\mathrm{SPO}_{2}(\mathrm{AMS}+72.0 \pm 2.4$ vs AMS -80.8 $\pm 2.8, P<.05)$. MAP was not different between groups on 
any intervention. During SB, CBFv was $75.4 \pm 8.1$ in AMS and elevated to $90.9 \pm 3.1$ in $\mathrm{AMS}+(P<.05)$. In both $\mathrm{AMS}+$ and AMS - , HVE led to elevation in $\mathrm{SPO}_{2}(\mathrm{AMS}+$ $81.1 \pm 3.0$ vs $\mathrm{AMS}-90.5 \pm 1.4, P<.05)$ and lower $\mathrm{CBFv}$ (AMS + $75.5 \pm 5.5$ vs AMS $-52.2 \pm 6.1$ ), but did not resolve the disparity between them. With HVE, AMS + CBFv dropped $18 \%$ compared to $30 \%$ in AMS $-(P<.05)$; the opposite was observed on $\mathrm{SB}-\mathrm{O}_{2}: \mathrm{AMS}+\mathrm{CBFv}$ dropped $30 \%$ compared to only $18 \%$ in $\mathrm{AMS}-(P<.05)$.

Conclusions. - The cerebral hemodynamic responses to lowered $\mathrm{CO}_{2}$ and elevated $\mathrm{O}_{2}$ were opposite between AMS+ and AMS - subjects. These findings suggest an important role for altered cerebral hemodynamics in the pathophysiology of AMS.

Robert C. Roach, $\mathrm{PhD}$ Denver, CO, USA

Ian J. C. MacCormick, MD Edinburgh, Midlothian, Scotland

Roger Thompson, MD Luc Bernardi, MD Gaia Casucci, MD Pavia, Italy

C. Matthew Kinsey, MD Boston, MA, USA 